\title{
BMJ Open Enhancing exercise tolerance and physical activity in COPD with combined pharmacological and non-pharmacological interventions: PHYSACTO randomised, placebo-controlled study design
}

\author{
Thierry Troosters, ${ }^{1}$ Jean Bourbeau, ${ }^{2}$ François Maltais, ${ }^{3}$ Nancy Leidy, ${ }^{4}$ \\ Damijan Erzen, ${ }^{5}$ Dorothy De Sousa, ${ }^{6}$ Lawrence Korducki, ${ }^{7}$ Alan Hamilton ${ }^{6}$
}

To cite: Troosters $T$,

Bourbeau J, Maltais F, et al. Enhancing exercise tolerance and physical activity in COPD with combined

pharmacological and non-pharmacological interventions: PHYSACTO randomised placebo-controlled study design. BMJ Open 2016;6 e010106. doi:10.1136/ bmjopen-2015-010106

- Prepublication history and additional material is available. To view please visit the journal (http://dx.doi.org/ 10.1136/bmjopen-2015010106).

Received 25 September 2015 Revised 29 January 2016 Accepted 1 February 2016

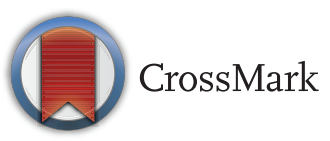

For numbered affiliations see end of article.

Correspondence to

Dr Thierry Troosters; Thierry Troosters@med.kuleuven.be

\section{ABSTRACT}

Introduction: Chronic obstructive pulmonary disease (COPD) is associated with exercise limitation and physical inactivity, which are believed to have significant long-term negative health consequences for patients. While a number of COPD treatments and exercise training programmes increase exercise capacity, there is limited evidence for their effects on physical activity levels, with no clear association between exercise capacity and physical activity in clinical trials. Physical activity depends on a number of behaviour, environmental and physiological factors. We describe the design of the PHYSACTO trial, which is investigating the effects of bronchodilators, either alone or with exercise training, in combination with a standardised behaviour-change self-management programme, on exercise capacity and physical activity in patients with COPD. It is hypothesised that bronchodilators in conjunction with a behaviourchange self-management programme will improve physical activity and that this effect will be amplified by the addition of exercise training.

Methods and analysis: Patients are being recruited from 34 sites in Australia, New Zealand, the USA, Canada and Europe. Patients receiving a multicomponent intervention designed to support behaviour change related to physical activity are randomised to four treatment arms: placebo, tiotropium, tiotropium+olodaterol, and tiotropium +olodaterol+exercise training. The primary outcome is improvement in exercise capacity after 8 weeks, measured by endurance time during a shuttle walk test. The secondary outcome is improvement in physical activity, including objective accelerometer assessment and patient-reported functioning using the Functional Performance Inventory-Short Form and the novel hybrid PROactive instrument. Additionally, the influence of moderating variables (ie, factors influencing a patient's choice to be physically active) on increases in physical activity is also explored.
Strengths and limitations of this study

- One strength of this study is that we are investigating the impact of a multimodal intervention (pharmacotherapy, exercise training and behaviour programme) on exercise capacity and physical activity.

- Another strength is the use of a behaviour change intervention combined with bronchodilators and exercise training in a multicentre, multinational study to encourage physical activity.

- We also measure physical activity using both accelerometers and patient-reported measures.

- A potential limitation to this work is that the study is only partially double-blinded as it is not possible to implement blinding in the exercise training intervention; this will be taken into account when data are analysed.

- A further limitation is that there is no control group that is not receiving the behaviour change intervention, so the effects of the behaviour change intervention alone cannot be singled out.

Ethics and dissemination: The study has been approved by the relevant Institutional Review Boards, Independent Ethics Committee and Competent Authority according to national and international regulations. The findings of the trial will be disseminated through relevant peer-reviewed journals and international conference presentations.

Trial registration number: NCT02085161.

\section{INTRODUCTION}

Chronic obstructive pulmonary disease (COPD) is associated with low levels of physical activity in daily life, starting early in the 
course of the disease. ${ }^{1}$ As breathlessness during activity worsens, patients may avoid physical activity, thereby causing muscle deconditioning and decreased exercise capacity, which further encourage a sedentary lifestyle. $^{23}$

Higher levels of physical activity in patients with COPD are associated with fewer symptoms, better health-related quality of life and a lower frequency of hospitalisations, exacerbations and mortality. ${ }^{4-7}$ In addition, it is suggested that there is an inverse relationship between activity level and rate of lung function decline. ${ }^{3}{ }^{8}$ However, it is difficult to establish a causal relationship with these outcomes: as disease severity increases, physical activity is likely to decrease. ${ }^{9}$

Improving exercise capacity and increasing physical activity levels are different yet related goals in COPD therapy. ${ }^{10}$ Exercise capacity is the amount of exercise patients are physically capable of doing, while physical activity reflects the amount of activity and the difficulty patients have in their activities in daily life. The European Respiratory Society statement on physical activity highlights the need for further research into physical activity in COPD. ${ }^{3}$

Exercise capacity can be directly measured in the clinic. A number of drugs that improve airflow have been shown to increase exercise capacity in COPD, though effect sizes vary between trials. ${ }^{1-15}$ Various types of exercise training also increase exercise capacity. ${ }^{16-20}$ One mechanism through which exercise training improves exercise capacity is by improving skeletal muscle function; ${ }^{21} 22$ this occurs when training intensity is high enough to provide an overload stimulus on the skeletal muscle. ${ }^{23}$ The combination of pulmonary rehabilitation with bronchodilator therapy increases exercise capacity to a greater extent than either component alone, ${ }^{24}$ and guidelines recommend that optimal bronchodilator therapy should be given prior to exercise training to enhance performance in and outside the training programme. ${ }^{25}$

A clear association in clinical trials between exercise capacity and physical activity levels has yet to be shown, ${ }^{26}$ with little and conflicting evidence from randomised controlled interventional studies demonstrating improvements in physical activity with pharmacological treatments (table 1) (range 4-11\% increase). ${ }^{7}{ }^{13} 27-29$ In the
PHYSACTO trial, it is assumed that improvement of exercise capacity is a prerequisite for increasing physical activity levels for the included interventions (bronchodilator therapy and exercise training) and, for this reason, exercise capacity is the primary end point of the study. It is becoming ever more apparent that increasing physical activity necessitates a change in patient behaviour. Consistent with this, activity counselling with direct feedback methods has been shown to improve physical activity levels in patients with $\mathrm{COPD},{ }^{30-33}$ as long as the intervention was maintained. ${ }^{34}$ The importance of behaviour change is reflected in the PHYSACTO trial by the inclusion of a standardised behaviour-change programme that is implemented in all treatment arms. This is described in the parallel publication by Bourbeau et al. $^{35}$

It is also important to understand the experience of physical activity from the patient's perspective. The Functional Performance Inventory-Short Form (FPI-SF) is used to capture patient report of the extent and difficulty with which patients perform daily activities, including those related to personal care, family and social aspects of life that people with COPD consider important. ${ }^{36}{ }^{37}$ In addition, a new approach to integrating quantity and quality of physical activity has been developed using a patientderived concept, ${ }^{38}$ through the European Innovative Medicines Initiative project, PROactive (http://www. proactivecopd.com). ${ }^{39}$ The instrument consists of questionnaire items and information from activity monitors, and has been developed to capture the patient's experience of physical activity in two domains: amount of activity and difficulties with physical activity.

\section{OBJECTIVES}

The first objective of the PHYSACTO trial is to confirm that long-acting muscarinic antagonist (LAMA) therapy and LAMA/long-acting $\beta_{2}$-agonist (LABA) combined therapy, alone or with exercise training, improve exercise endurance capacity.

The second objective is to evaluate the extent to which pharmacotherapy alone and in combination with exercise training can enhance the effects of a behaviourchange intervention that aims to enhance physical activity levels. The amount of physical activity is

Table 1 Changes in step count in double-blind studies investigating the effect of pharmacotherapy on physical activity

\begin{tabular}{|c|c|c|c|c|c|c|}
\hline Group & Intervention & $\begin{array}{l}\text { Activity } \\
\text { sensor }\end{array}$ & Study design & $\begin{array}{l}\text { Duration, } \\
\text { weeks }\end{array}$ & $\begin{array}{l}\text { Baseline } \\
\text { steps } \\
\text { per day }\end{array}$ & $\begin{array}{l}\text { Difference from } \\
\text { placebo in step count, } \\
\text { steps per day }\end{array}$ \\
\hline Beeh et $a^{77}$ & Aclidinium & SenseWear & Cros & 3 & 7030 & $459(7 \%)$ \\
\hline Watz et $a p^{8}$ & Indacaterol & SenseWear & Crossover & 3 & $6618^{*}$ & $722(11 \%)$ \\
\hline Troosters et $a f^{29}$ & Tiotropium & SenseWear & Parallel group & 24 & 6400 & $400(6 \%)$ \\
\hline O'Donnell et al ${ }^{13}$ & Indacaterol & SenseWear & Crossover & 3 & $46.2 \mathrm{~min}^{\star} \dagger$ & $1.9 \mathrm{~min} † \ddagger(4 \%)$ \\
\hline
\end{tabular}

${ }^{*}$ Data represent the placebo group to give a general idea of physical activity in the study population because baseline data are not available. †Data presented in physical activity time in min/day.

$\ddagger$ Difference from placebo. 
measured in this trial using validated triaxial accelerometers. In addition, patient-reported physical activity is assessed as both the perceived amount of activity and the perceived difficulty of physical activity. Hence, this is the first trial to explore whether increasing exercise capacity using non-pharmacological and pharmacological means, in combination with behaviour change, can also increase the amount of physical activity performed by patients with COPD.

The trial also explores how patient characteristics and other factors such as motivation and self-efficacy influence improvements in physical activity. An additional objective of the trial is to further validate the PROactive instrument by testing its sensitivity to interventions in a randomised clinical trial.

\section{METHODS}

\section{Study design}

PHYSACTO is a randomised, partially double-blinded, placebo-controlled, parallel-group trial (NCT02085161) being conducted at 34 sites in Australia, New Zealand, the USA, Canada and Europe consisting of 17 academic institutes, 15 secondary care and 5 primary care centres. A full list of sites can be obtained at https://clinicaltrials.gov. Patients are randomised in equal ratio to four groups: placebo, tiotropium $5 \mu \mathrm{g}$ once daily, tiotropium +olodaterol $5 / 5 \mu \mathrm{g}$ once daily, and tiotropium+olodaterol $5 / 5 \mu \mathrm{g}$ once daily with exercise training; all groups will receive 12 weeks of a behaviour-change selfmanagement programme (figure 1). Medications (active treatment as well as placebo) are administered using the Respimat inhaler (Boehringer Ingelheim, Ingelheim am Rhein, Germany) for 12 weeks and exercise training is provided for the first 8 weeks of treatment. Patients are permitted to continue with inhaled corticosteroids but not LAMAs, LABAs or short-acting muscarinic antagonists during the trial, and all are provided with openlabel salbutamol for rescue medication use throughout the trial. Acute exacerbations can be treated as medically necessary. This is similar to the approach taken for other LAMA/LABA development programmes, such as umeclidinium+vilanterol, which have included several placebo-controlled studies of 26-52 week duration, ${ }^{40} 41$ and this is in accordance with statements from the Food and Drug Administration. ${ }^{42}$

\section{Inclusion/exclusion criteria}

Patients with COPD are included if they have postbronchodilator forced expiratory volume in $1 \mathrm{~s}\left(\mathrm{FEV}_{1}\right)$ $\geq 30 \%$ and $<80 \%$ of predicted normal (Global initiative for chronic Obstructive Lung Disease 2-3) and did not experience acute exacerbations in the month prior to the study, postbronchodilator $\mathrm{FEV}_{1} /$ forced vital capacity $<70 \%$, are aged $\geq 40$ years and $\leq 75$ years, and have a smoking history of $>10$ pack-years. Patients are excluded if they have a significant disease other than COPD, a history of asthma, clinically relevant abnormal baseline haematology, blood chemistry or urinalysis, or conditions excluding them from exercise.

Willingness to increase physical activity is not an inclusion criterion but patients have to be willing to take part in the 8-week exercise training programme ( 1 in 4 chance of being randomised to the treatment arm that includes exercise training) and take part in the behaviour-change self-management programme.

\section{Behaviour change and exercise training}

All patients participate in a 12-week behaviour-change self-management programme adapted from the 'Living Well with COPD' programme, ${ }^{43}$ which consists of a selfmanagement educational programme, support from a Site Case Manager (SCM) and evaluation using standardised questionnaires to monitor patients' motivation, confidence and movement through stages of change. This is described in detail in Bourbeau et al. ${ }^{35}$ Patients also receive a step-counter (OMRON HJ-321) to track their physical activity in real time towards achievement of their step-counter goals.

To ensure consistency across sites, a global training team provides face-to-face training to SCMs from each site

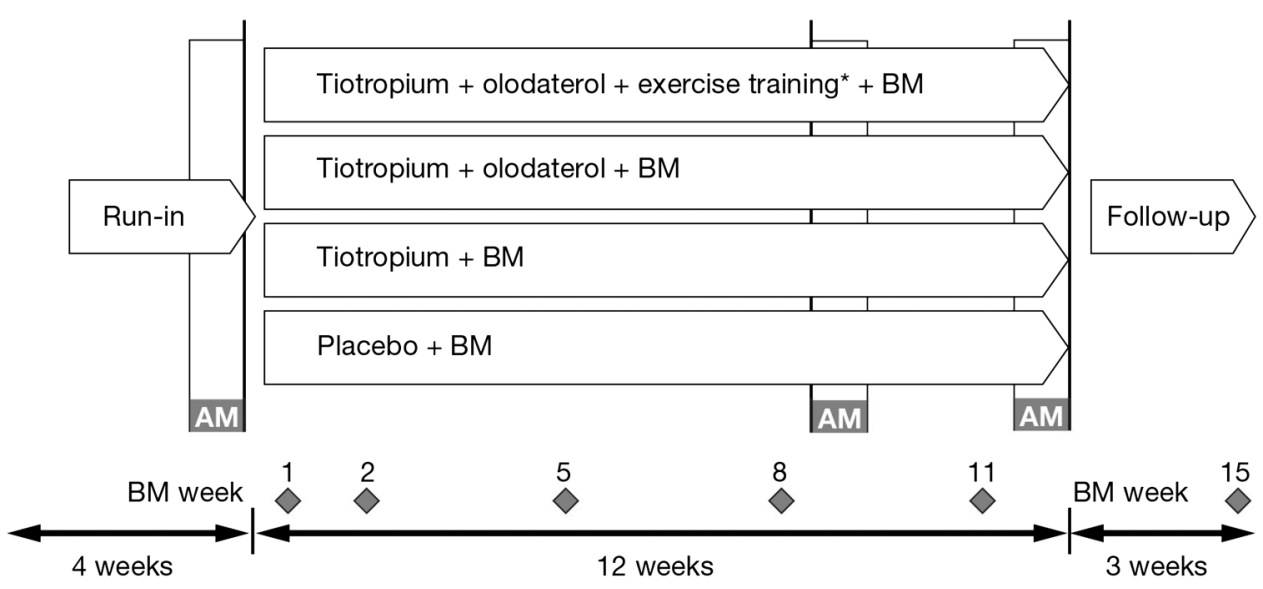

Figure 1 PHYSACTO trial design. *Eight weeks only. AM, activity monitoring, 1 week; BM, behaviour modification at weeks 1 , 2, 5, 8, 11 and 15. 
and ensures that the behaviour-change self-management programme is implemented in a standardised fashion, as described in detail in Bourbeau et al. ${ }^{35} \mathrm{SCMs}$ also receive a comprehensive reference guide, and sessions are audiotaped and sent to a Country Case Manager for centralised review to ensure quality control and consistency of sessions.

Exercise training is provided only to patients in one of the tiotropium+olodaterol $5 / 5 \mu \mathrm{g}$ groups and consists of 8 weeks of high-intensity whole-body exercise training, as well as lower limb resistance training according to international standards. ${ }^{21} 44$ It is conducted three times per week in an outpatient rehabilitation facility and training logs are kept. Training intensity is high, and is increased gradually based on symptom scores for fatigue and dyspnoea. A global trainer is responsible for ensuring that exercise training and testing are carried out in a consistent way across sites.

\section{End points}

The primary end point is endurance time during an endurance shuttle walk test $(\mathrm{ESWT})^{45}$ to symptom limitation after 8 weeks of therapy (to reflect the 8-week duration of the exercise training programme). ESWT is performed at $85 \%$ of maximum oxygen consumption $\left(\mathrm{VO}_{2}\right.$ peak) as predicted from an incremental shuttle walk test conducted at visit 1 . A practice ESWT will be provided during the screening period prior to a baseline ESWT conducted at a walk speed corresponding to $85 \%$ of $\mathrm{VO}_{2}$ peak. Both 8-week and 12-week ESWT will be done $2 \mathrm{~h}(+/-15 \mathrm{~min})$ after inhalation of the study medication. Table 2 shows the secondary, safety and exploratory end points.

In order to gain an accurate, objective assessment of patient physical activity levels, walking intensity, walking time and number of steps are measured in all patients using an activity monitor. ${ }^{3}$ An activity monitor (MoveMonitor Dynaport, McRoberts, The Hague, the Netherlands) is worn by patients while they are awake every day for 1 week at three points in the trial: 1 week during the screening period; the week prior to the week 9 visit; and the week prior to the week 12 visit. Patients complete the FPI-SF questionnaire at clinic visits during screening and at weeks 9 and 12. They also complete the daily PROactive questionnaire on a personal digital assistant on the days they

Table 2 Trial end points and safety assessments

\begin{tabular}{|c|c|c|}
\hline & End point & Time \\
\hline Primary end point & $\begin{array}{l}\text { Endurance time during ESWT to symptom limitation at } 85 \% \text { of } \\
\mathrm{VO}_{2} \text { peak }\end{array}$ & Week 8 \\
\hline Secondary end point & Average daily walking time measured by activity monitor & Week prior to week 12 visit \\
\hline Secondary end point & Average daily walking intensity measured by activity monitor & Week prior to week 12 visit \\
\hline Secondary end point & Perceived ease/difficulty with daily activities assessed by FPI-SF & Week 12 \\
\hline Secondary end point & $\begin{array}{l}\text { Endurance time during ESWT to symptom limitation at } 85 \% \text { of } \\
\mathrm{VO}_{2} \text { peak }\end{array}$ & Week 12 \\
\hline Secondary end point & $1 \mathrm{~h}$ postdose $\mathrm{FEV}_{1}$ & Week 8 \\
\hline Secondary end point & $1 \mathrm{~h}$ postdose forced vital capacity & Week 8 \\
\hline Secondary end point & Resting inspiratory capacity measured at $1.5 \mathrm{~h}$ postdose & Week 8 \\
\hline Further efficacy end point & PROactive daily difficulties domain and amount domain scores & Week 12/weeks 8, 12 \\
\hline Further efficacy end point & Distance measured during 6MWT & Weeks 8, 12 \\
\hline Further efficacy end point & $\begin{array}{l}\text { Intensity of breathing discomfort during 6MWT: pre-exercise, } \\
\text { during exercise and at end-exercise }\end{array}$ & Weeks 8,12 \\
\hline Further efficacy end point & $\begin{array}{l}\text { Intensity of breathing discomfort during ESWT: pre-exercise, } \\
\text { during exercise and at end-exercise }\end{array}$ & Weeks 8,12 \\
\hline Further efficacy end point & $\begin{array}{l}\text { Average number of steps per day measured by the activity } \\
\text { monitor }\end{array}$ & $\begin{array}{l}\text { Week preceding week } 9 \text { visit, } \\
\text { week preceding week } 12 \text { visit }\end{array}$ \\
\hline Further efficacy end point & Health status measured by SGRQ & Weeks 9, 12 \\
\hline Safety assessments & Heart rate and blood pressure in conjunction with spirometry & Weeks $1,8,12$ \\
\hline Safety assessments & $\begin{array}{l}\text { Heart rate, oxygen saturation and blood pressure in conjunction } \\
\text { with exercise testing }\end{array}$ & Weeks 8,12 \\
\hline Safety assessments & $\begin{array}{l}\text { All adverse events (including physical examination) until end of } \\
\text { study }\end{array}$ & Throughout \\
\hline Other outcomes & $\begin{array}{l}\text { A multivariate regression analysis of the following factors that may } \\
\text { affect activity: motivation, self-efficacy, cognitive function, } \\
\text { depression, anxiety, baseline level of activity, } 6 \mathrm{MWT} \text { distance, } \\
\text { BMI, sex, age, waist circumference, rescue medication use, and } \\
\text { external and internal barriers (eg, environmental/seasonal, social, } \\
\text { economic) }\end{array}$ & \\
\hline
\end{tabular}


wear the activity monitor. Pulmonary function testing will be conducted prior to ESWT according to the American Thoracic Society/European Respiratory Society guidelines ${ }^{46}$ at screening to determine eligibility, prior to randomisation to determine baseline values, and at clinic visits on the first day of treatment and after 8 and 12 weeks of treatment.

\section{Safety}

Safety assessments include heart rate and blood pressure in conjunction with spirometry, and heart rate, blood pressure and oxygen saturation in conjunction with exercise testing. Physical examinations are performed and adverse events including exacerbations are monitored carefully throughout the trial.

\section{Randomisation}

Randomisation is performed using a pseudorandom number generator and block randomisation is used to achieve balanced allocation. Patients are assigned to treatment arms using a web-based and telephone-based response system, and provided with numbered medication boxes. A double-blind design is used for the groups receiving placebo+behaviour modification, tiotropium monotherapy+behaviour modification, and tiotropium +olodaterol+behaviour modification. It is not possible to blind the participants or staff against the group receiving tiotropium+olodaterol+exercise training+behaviour modification. This is because placebo combined with exercise training is not in accordance with current recommendations.

\section{Sample size}

Using an estimate of SD for a $\log _{10}$-transformed endurance time of $0.213 \mathrm{~s}$, a sample size of 75 patients per treatment group will provide a power of $80-90 \%$ to detect an improvement of $25-30 \%$ in endurance time between tiotropium+olodaterol $5 / 5 \mu \mathrm{g}$ versus placebo from ESWT. No sample size calculation was performed for activity variables.

\section{Statistical analysis}

The primary end point of endurance time during ESWT will be $\log _{10}$-transformed to correct for skewness and an analysis of covariance model, including treatment as a fixed categorical end point and $\log _{10}$-baseline endurance time as a continuous covariate, will be used. A similar analysis of covariance model will be used for the secondary end points. The influence of a number of moderating variables measured at baseline (motivation, self-efficacy, cognitive function, depression, anxiety, baseline level of physical activity, 6 min walk test distance, body mass index, sex, age, rescue medication use, and external and internal barriers (eg, environmental/seasonal factors and social and economic factors)) on increase in the amount of physical activity and perceived difficulties during physical activity will also be explored.

\section{Data dissemination}

The studies are to be carried out in accordance with the principles of the Declaration of Helsinki and the International Conference on Harmonisation Harmonised Tripartite Guideline for Good Clinical Practice, and written informed consent will be obtained from each patient.

\section{DISCUSSION}

The PHYSACTO trial is being performed to investigate how pharmacological interventions in combination with exercise training can improve exercise capacity. The study will also investigate to what extent pharmacotherapy, alone and combined with exercise training, is an enabler for effective physical activity behaviour change induced by a self-management programme aiming to enhance physical activity. To allow investigation of these questions, multiple interventions are included in the trial, where the addition of each intervention (the addition of a bronchodilator to placebo, then a second bronchodilator, etc) is expected to provide an incremental improvement in exercise capacity and, possibly with the aid of a behaviour-change programme, an incremental improvement in physical activity. This will also allow evaluation of the responsiveness of the instruments being used to measure physical activity, in particular the PROactive tool.

In order to evaluate effect size, it was necessary to include a placebo arm; this also allows the impact of pharmacotherapy on top of the behaviour intervention to be determined. A placebo arm was considered acceptable given that the population has moderate-to-severe COPD (patients with very severe COPD are not eligible), the trial duration is 3 months and patients are seen regularly (as part of the behaviour-change programme). Patients also continue with inhaled corticosteroid therapy (if taken at baseline), are provided with rescue medication and withdrawal is considered if there is significant clinical deterioration (ie, repeated exacerbations or clinical deterioration requiring maintenance therapy not allowed as per protocol).

It is becoming increasingly clear that COPD disease management needs to transition from an acute-care model to a patient-centred, integrated, chronic care model, ${ }^{47}$ where different types of intervention are combined to serve individual patient needs. It is expected that improvements in exercise capacity in this trial will be directly caused by bronchodilators and exercise training. A behaviour-change programme was included to support the translation of increased exercise capacity to increased physical activity. By providing a combination of optimal pharmacotherapy and interventions directed at improving the next limiting factor (ie, skeletal muscle) and encouraging effective behaviour change, this trial provides a view on modern integrated COPD management. Rolling out such a complex intervention does not come without challenges. 
Exercise training and a behaviour-change programme in a large multinational study must be standardised and conducted according to guidelines. This can be particularly challenging in different cultures with varying health beliefs and communication styles and previous rehabilitation experience. The purpose of the global trainer for the exercise training and testing, as well as that of the global training team for the behaviourchange programme, is to optimise consistency across sites. This is described in more detail in another manuscript. ${ }^{35}$

Use of the ESWT, activity monitors and patientreported outcomes provides the opportunity to first confirm that the interventions being tested increase exercise capacity in this trial, and to then identify whether improvements in exercise capacity are translated into increased physical activity. The ESWT was selected as the measure of exercise capacity rather than constant work-rate cycle ergometry because it is considered to be more representative of the activities that patients perform in everyday life, requires less equipment and has adequate measurement properties. ${ }^{45}$

In this study, exercise training is provided for 8 weeks while all other interventions are provided for 12 weeks. Physical activity is measured at 12 weeks to avoid exercise training confounding the measurement of physical activity, and also to ensure that there is sufficient time for patients to resume 'normal', and hopefully improved, activity patterns after exercise training has ended. Exercise capacity is measured at 8 weeks, at the end of the exercise training programme. Eight weeks have been shown to be long enough to increase exercise capacity with exercise training, bronchodilator therapy and their combinations. ${ }^{111216} 19$

Exercise programmes alone may or may not change individual patient activity levels. Between exercise capacity and physical activity lie a host of moderating variables that influence a patient's choice to perform physical activity ${ }^{48}{ }^{49}$ Since the choice to utilise increased exercise capacity will vary between individuals, one might expect the direct statistical relationship between physical training programmes and physical activity levels in a clinical trial population to be weak, and the indirect, moderating effects to be stronger.

Therefore, it is important to acknowledge the influence of physiological, genetic, psychosocial and environmental factors on a patient's ability, motivation and confidence to increase physical activity. ${ }^{4} \quad 50 \quad 51$ The interactions between patient characteristics (such as baseline activity level and exercise capacity, anxiety and age) and physical activity levels are not fully understood, ${ }^{7}$ and longitudinal interventional studies allowing for a more direct interpretation of cause and effect are lacking. It is also known that external factors such as climate, altitude and season affect physical activity levels. ${ }^{3}{ }^{52}$ The PHYSACTO trial will explore the extent to which a number of postulated characteristics influence the increase in amount of physical activity and perceived difficulties with physical activity demonstrated in this trial.

There are potential limitations of the study. It is only partially double-blinded, as it is not possible to blind the group receiving exercise training; interpretation of the data from this group will need to take into account the potential biases associated with the knowledge of treatment group. We did not include a placebo group without the behaviour-change programme or a run-in period with the behaviour-change programme and standard pharmacotherapy in the study design, so the effects of the behaviour-change self-management programme alone cannot be assessed in the present study. Rather, this study is designed to test whether the addition of more optimal pharmacotherapy (tiotropium or tiotropium+olodaterol) alone or in combination with exercise training can enhance the effects of the behaviour-change programme. Depending on the results of this study, further research will most likely be warranted to address the best approach to support behaviour change.

Overall, it is hoped that this study will increase our understanding of how exercise capacity can be increased in patients with COPD and how this improvement can be translated into increased physical activity levels. The trial also prepares the field for comprehensive COPD patient management, including complementary pharmacological and non-pharmacological treatments.

\section{Author affiliations}

${ }^{1}$ Department of Rehabilitation Sciences, Pulmonary Rehabilitation and Respiratory Division, University Hospital Leuven, KU Leuven, Leuven, Belgium

${ }^{2}$ Respiratory Epidemiology Clinical Research Unit, Research Institute of the McGill University Health Centre, Montreal, Quebec, Canada

${ }^{3}$ Centre de Recherche, Institut Universitaire de Cardiologie et de Pneumologie de Quebec, Université Laval, Quebec, Canada

${ }^{4}$ Evidera, Bethesda, Maryland, USA

${ }^{5}$ Boehringer Ingelheim Pharma GmbH \& Co. KG, Biberach an der Riss, Germany

${ }^{6}$ Boehringer Ingelheim (Canada) Ltd, Burlington, Ontario, Canada ${ }^{7}$ Boehringer Ingelheim Pharmaceuticals Inc., Ridgefield, Connecticut, USA

Contributors TT and JB are the principal investigators in the PHYSACTO trial. FM and NL are part of the protocol steering committee. JB provided guidance for the design of the behaviour modification programme. TT and FM provided expertise in exercise training and TT provided guidance on the implementation of the PROactive tool. NL was involved in the protocol design and specifically provided expertise in the patient-reported outcomes and perception scales. LK is the statistician for the trial. DE, DDS and AH were involved in the design of the study and are involved in the implementation of the study. The authors meet criteria for authorship as recommended by the International Committee of Medical Journal Editors. They take full responsibility for the scope, direction, content of, and editorial decisions relating to, the manuscript, were involved at all stages of development and have approved the submitted manuscript. The authors received no compensation related to the development of the manuscript.

Funding This work was supported by Boehringer Ingelheim Pharma GmbH \& Co. KG. Medical writing assistance was provided by Claire Scofield of Complete HealthVizion, which was contracted and compensated by Boehringer Pharma GmbH \& Co. KG. The PROactive project is funded by the IMI-JU (\#115011). The PHYSACTO trial is an in-kind contribution of the sponsor Boehringer Ingelheim to the PROactive project. In this project, the innovative patient-reported outcome tool and the activity monitor were developed and validated. 
Competing interests TT has received grants from the Innovative Medicines Initiative Joint Undertaking and speaker fees from Boehringer Ingelheim and GlaxoSmithKline. JB has received grants from the Canadian Institute of Health Research R\&D collaborative programme (AstraZeneca, Boehringer Ingelheim, GlaxoSmithKline, Merck, Nycomed, Novartis), the Canadian Respiratory Research Network, the Respiratory Health Network of the FRQS and the Research Institute of the MUHC. FM has received grants from Boehringer Ingelheim, GlaxoSmithKline, AstraZeneca, Nycomed and Pfizer, personal fees from Boehringer Ingelheim, GlaxoSmithKline and Novartis, and other financial support from GlaxoSmithKline. NL is employed by Evidera, a healthcare research firm that provides consulting and other research services to pharmaceutical and other organisations including the study sponsor. DE, DDS, LK and AH are employees of Boehringer Ingelheim. DE, DDS and AH are employees of the sponsor and were involved in the design, implementation and interpretation of the study results, together with the investigators.

\section{Patient consent Obtained.}

Ethics approval The protocol was approved on 19/02/2014 by the Southern Adelaide Clinical Human Research Ethics Committee (11.14HREC/14/SAC/19). All other participating centres subsequently approved the study.

Provenance and peer review Not commissioned; externally peer reviewed.

Data sharing statement The findings of the trial will be disseminated through relevant peer-reviewed journals and international conference presentations. This study design is reported in accordance with the Standard Protocol Items: Recommendations for Interventional Trials (SPIRIT) guidelines. ${ }^{53}$ Further details are included in the supplementary material available online, including items from the World Health Organization Trial Registration Data Set (see online supplementary table S1), a list of further end points (see online supplementary table S2) and a description of the morning and bedtime diaries (see online supplementary tables S3 and S4).

Open Access This is an Open Access article distributed in accordance with the Creative Commons Attribution Non Commercial (CC BY-NC 4.0) license, which permits others to distribute, remix, adapt, build upon this work noncommercially, and license their derivative works on different terms, provided the original work is properly cited and the use is non-commercial. See: http:// creativecommons.org/licenses/by-nc/4.0/

\section{REFERENCES}

1. van Remoortel $\mathrm{H}$, Hornikx $\mathrm{M}$, Demeyer $\mathrm{H}$, et al. Daily physical activity in subjects with newly diagnosed COPD. Thorax 2013;68:962-3.

2. Troosters $\mathrm{T}$, van der Molen $\mathrm{T}$, Polkey $\mathrm{M}$, et al. Improving physical activity in COPD: towards a new paradigm. Respir Res 2013;14:115

3. Watz H, Pitta F, Rochester CL, et al. An official European Respiratory Society statement on physical activity in COPD. Eur Respir J 2014;44:1521-37.

4. Leidy NK, Kimel M, Ajagbe L, et al. Designing trials of behavioral interventions to increase physical activity in patients with COPD: insights from the chronic disease literature. Respir Med 2014:108:472-81.

5. ZuWallack R, Esteban C. Understanding the impact of physical activity in COPD outcomes: moving forward. Eur Respir $J$ 2014:44:1107-9.

6. Garcia-Aymerich J, Lange P, Benet M, et al. Regular physical activity reduces hospital admission and mortality in chronic obstructive pulmonary disease: a population based cohort study. Thorax 2006;61:772-8.

7. Gimeno-Santos E, Frei A, Steurer-Stey C, et al. Determinants and outcomes of physical activity in patients with COPD: a systematic review. Thorax 2014;69:731-9.

8. Garcia-Aymerich J, Lange $\mathrm{P}$, Benet $\mathrm{M}$, et al. Regular physical activity modifies smoking-related lung function decline and reduces risk of chronic obstructive pulmonary disease. A population-based cohort study. Am J Respir Crit Care Med 2007;175:458-63.

9. Waschki B, Kirsten AM, Holz O, et al. Disease progression and changes in physical activity in patients with chronic obstructive pulmonary disease. Am J Respir Crit Care Med 2015;192:295-306.

10. Global Initiative for Chronic Obstructive Lung Disease. Global strategy for the diagnosis, management, and prevention of chronic obstructive pulmonary disease. Updated 2014. 2014. http://www.
goldcopd.org/uploads/users/files/GOLD_Report2014_Feb07.pdf (accessed 22 Jul 2015)

11. Beeh KM, Singh D, Di Scala L, et al. Once-daily NVA237 improves exercise tolerance from the first dose in patients with COPD: the GLOW3 trial. Int J Chron Obstruct Pulmon Dis 2012;7:503-13.

12. Maltais F, Celli B, Casaburi R, et al. Aclidinium bromide improves exercise endurance and lung hyperinflation in patients with moderate to severe COPD. Respir Med 2011;105:580-7.

13. O'Donnell DE, Casaburi R, Vincken W, et al. Effect of indacaterol on exercise endurance and lung hyperinflation in COPD. Respir Med 2011;105:1030-6.

14. Maltais F, Hamilton A, Marciniuk D, et al. Improvements in symptom-limited exercise performance over $8 \mathrm{~h}$ with once-daily tiotropium in patients with COPD. Chest 2005;128:1168-78.

15. O'Donnell DE, Voduc N, Fitzpatrick $M$, et al. Effect of salmeterol on the ventilatory response to exercise in chronic obstructive pulmonary disease. Eur Respir J 2004;24:86-94.

16. McCarthy B, Casey D, Devane D, et al. Pulmonary rehabilitation for chronic obstructive pulmonary disease. Cochrane Database Syst Rev 2015;(2):CD003793.

17. Klijn $\mathrm{P}$, van Keimpema $\mathrm{A}$, Legemaat $\mathrm{M}$, et al. Nonlinear exercise training in advanced chronic obstructive pulmonary disease is superior to traditional exercise training. A randomized trial. $A m \mathrm{~J}$ Respir Crit Care Med 2013;188:193-200.

18. McNamara RJ, McKeough ZJ, McKenzie DK, et al. Water-based exercise in COPD with physical comorbidities: a randomised controlled trial. Eur Respir J 2013;41:1284-91.

19. Ringbaek T, Brøndum E, Martinez G, et al. Rehabilitation in COPD: the long-term effect of a supervised 7-week program succeeded by a self-monitored walking program. Chron Respir Dis 2008;5: 75-80.

20. Dias FD, Sampaio LM, da Silva GA, et al. Home-based pulmonary rehabilitation in patients with chronic obstructive pulmonary disease: a randomized clinical trial. Int $J$ Chron Obstruct Pulmon Dis 2013;8:537-44.

21. Maltais F, Decramer M, Casaburi R, et al. An official American Thoracic Society/European Respiratory Society statement: update on limb muscle dysfunction in chronic obstructive pulmonary disease. Am J Respir Crit Care Med 2014;189:e15-62.

22. Pepin V, Saey D, Laviolette L, et al. Exercise capacity in chronic obstructive pulmonary disease: mechanisms of limitation. COPD 2007;4:195-204.

23. Burtin C, Saey D, Saglam M, et al. Effectiveness of exercise training in patients with COPD: the role of muscle fatigue. Eur Respir $J$ 2012;40:338-44.

24. Casaburi R, Kukafka D, Cooper CB, et al. Improvement in exercise tolerance with the combination of tiotropium and pulmonary rehabilitation in patients with COPD. Chest 2005;127:809-17.

25. Nici L, Donner C, Wouters E, et al. American Thoracic Society/ European Respiratory Society statement on pulmonary rehabilitation. Am J Respir Crit Care Med 2006;173:1390-413.

26. Troosters T. Physical inactivity in patients with COPD: the next step is ... action. Prim Care Respir J 2013:22:391-2.

27. Beeh KM, Watz $\mathrm{H}$, Puente-Maestu L, et al. Aclidinium improves exercise endurance, dyspnea, lung hyperinflation, and physical activity in patients with COPD: a randomized, placebo-controlled, crossover trial. BMC Pulm Med 2014;14:209.

28. Watz H, Krippner F, Kirsten A, et al. Indacaterol improves lung hyperinflation and physical activity in patients with moderate chronic obstructive pulmonary disease-a randomized, multicenter, double-blind, placebo-controlled study. BMC Pulm Med 2014; $14: 158$.

29. Troosters T, Sciurba FC, Decramer M, et al. Tiotropium in patients with moderate COPD naive to maintenance therapy: a randomised placebo-controlled trial. NPJ Prim Care Respir Med 2014;24:14003.

30. Effing $\mathrm{T}$, Zielhuis $\mathrm{G}$, Kerstjens $\mathrm{H}$, et al. Community based physiotherapeutic exercise in COPD self-management: a randomised controlled trial. Respir Med 2011;105:418-26.

31. Hospes $\mathrm{G}$, Bossenbroek L, ten Hacken $\mathrm{NH}$, et al. Enhancement of daily physical activity increases physical fitness of outclinic COPD patients: results of an exercise counseling program. Patient Educ Couns 2009;75:274-8.

32. de Blok BMJ, de Greef MHG, ten Hacken NHT, et al. The effects of a lifestyle physical activity counseling program with feedback of a pedometer during pulmonary rehabilitation in patients with COPD: a pilot study. Patient Educ Couns 2006;61:48-55.

33. Mendoza L, Horta P. Espinoza J, et al. Pedometers to enhance physical activity in COPD: a randomised controlled trial. Eur Respir J 2015;45:347-54.

34. Altenburg WA, ten Hacken NHT, Bossenbroek $L$, et al. Short- and long-term effects of a physical activity counselling programme 
in COPD: a randomized controlled trial. Respir Med 2015;109:112-21.

35. Bourbeau J, Lavoie LK, Sedeno M, et al. Behaviour-change intervention in a multicentre, randomised, placebocontrolled COPD study: methodological considerations and implementation. BMJ Open 2016;6:e010109.

36. Leidy NK, Hamilton A, Becker K. Assessing patient report of function: content validity of the Functional Performance Inventory-Short Form (FPI-SF) in patients with chronic obstructive pulmonary disease (COPD). Int J Chron Obstruct Pulmon Dis 2012;7:543-54.

37. Leidy NK, Knebel A. In search of parsimony: reliability and validity of the Functional Performance Inventory-Short Form. Int J Chron Obstruct Pulmon Dis 2010;5:415-23.

38. Dobbels $F$, de Jong $C$, Drost $\mathrm{E}$, et al. The PROactive innovative conceptual framework on physical activity. Eur Respir $J$ 2014;44:1223-33.

39. Gimeno-Santos E, Raste Y, Demeyer H, et al. The PROactive instruments to measure physical activity in patients with chronic obstructive pulmonary disease. Eur Respir J 2015;46: 988-1000.

40. Blair HA, Deeks ED. Umeclidinium/vilanterol: a review of its use as maintenance therapy in adults with chronic obstructive pulmonary disease. Drugs 2015;75:61-74.

41. Prakash A, Babu KS, Morjaria JB. Profile of inhaled glycopyrronium bromide as monotherapy and in fixed-dose combination with indacaterol maleate for the treatment of COPD. Int J Chron Obstruct Pulmon Dis 2015;10:111-23.

42. US Food and Drug Administration. NDA 203-975: umeclidinium and vilanterol inhalation powder for the long-term, once-daily maintenance treatment of airflow obstruction in patients with chronic obstructive pulmonary disease (COPD). 2013. http://www.fda.gov/ downloads/AdvisoryCommittees/CommitteesMeetingMaterials/ Drugs/Pulmonary-AllergyDrugsAdvisoryCommittee/UCM367411.pdf (accessed 22 Jan 2016).
43. Living Well with COPD ${ }^{\mathrm{TM}}$. 2015. http://www.livingwellwithcopd.com/ en/home.html (accessed 12 Jan 2015).

44. Spruit MA, Singh SJ, Garvey C, et al. An official American Thoracic Society/European Respiratory Society statement: key concepts and advances in pulmonary rehabilitation. Am J Respir Crit Care Med 2013;188:e13-64

45. Holland AE, Spruit MA, Troosters T, et al. An official European Respiratory Society/American Thoracic Society technical standard: field walking tests in chronic respiratory disease. Eur Respir J 2014;44:1428-46.

46. Miller MR, Hankinson J, Brusasco V, et al. Standardisation of spirometry. Eur Respir J 2005;26:319-38.

47. Nici L, ZuWallack R, American Thoracic Society Subcommittee on Integrated Care of the COPD Patient. An official American Thoracic Society workshop report: the Integrated Care of The COPD Patient. Proc Am Thorac Soc 2012;9:9-18.

48. Leidy NK. Using functional status to assess treatment outcomes. Chest 1994;106:1645-6.

49. Stull DE, Leidy NK, Jones PW, et al. Measuring functional performance in patients with COPD: a discussion of patient-reported outcome measures. Curr Med Res Opin 2007;23:2655-65.

50. Bauman AE, Reis RS, Sallis JF, et al. Correlates of physical activity: why are some people physically active and others not? Lancet 2012;380:258-71.

51. Thorpe O, Kumar S, Johnston K. Barriers to and enablers of physical activity in patients with COPD following a hospital admission: a qualitative study. Int $J$ Chron Obstruct Pulmon Dis 2014;9:115-28.

52. Demeyer $\mathrm{H}$, Burtin $\mathrm{C}$, van Remoortel $\mathrm{H}$, et al. Standardizing the analysis of physical activity in patients with COPD following a pulmonary rehabilitation program. Chest 2014;146: 318-27.

53. Chan AW, Tetzlaff JM, Gotzsche PC, et al. SPIRIT 2013 explanation and elaboration: guidance for protocols of clinical trials. BMJ 2013;346:e7586. 